\title{
Do Corticosteroids Affect Prenatal Biophysical Parameters? A Systematic Review and Meta-analysis
}

\author{
Sanaz Musavi ${ }^{\circledR}$, Leila Nikniaz ${ }^{2}$, Hosein Hoseinifard ${ }^{3}$, Arezou Hamzehzadeh ${ }^{4}$, Shabnam Vazifekhah ${ }^{5 *}(\mathbb{0}$
}

\begin{abstract}
This systematic review and meta-analysis aimed to evaluate the effect of betamethasone and dexamethasone on biophysical profile (BPP) parameters. In addition, it was performed in 2017, using several databases such as PubMed/MEDLINE, Scopus, EMBASE, Cochrane library, ISI Web of science, Proquest, and Google scholar, along with Magiran SID and IranMedex. Eligible studies were selected by two reviewers and the outcomes of interest were extracted as well. Meta-analysis was done using the random effect model. Further, I-square statistic test was used for heterogeneity analysis and the presence of publication bias was also checked. At last, 12 studies were included and a random and fixed effect model was used for analysis. The pooled event rates were 4.5\% (95\% CI $=0.01-64.3, P=0.1), 76.8 \%$ (\% $95 \mathrm{CI}=33.5-95.6, P=0.21), 71.8 \%$ (\% $95 \mathrm{CI}=38.8-91.1, P=0.18), 70.9 \%(\% 95 \mathrm{CI}=38.4-90.5, P=0.20)$, and $92.3 \%$ (\%95 $\mathrm{CI}=76.0-97.8, \mathrm{P}<0.001)$ for the reduced amniotic fluid volume, baseline fetal heart rate reactivity, fetal breathing, fetal movement, and heart rate variability, respectively. In summary, a significant decrease was observed in heart rate variability following betamethasone and dexamethasone administration. However, further systematic reviews are necessary to differentiate steroid induced changes in the fetal BPP from those due to fetal compromise.

Keywords: Biophysical profile parameters, Betamethasone, Dexamethasone, Amniotic fluid volume, Fetal body movements, Breathing movements, Fetal heart rate reactivity
\end{abstract}

\section{Introduction}

Preterm labor is one of the most common reasons for the hospitalization of pregnant women which complicates $11.7 \%$ of pregnancies. (1) In general agreements with recommendations from the American College of Obstetricians and Gynecologists, women diagnosed with preterm labor $<34$ weeks of gestation are hospitalized, and a course of betamethasone is administered to reduce neonatal morbidity and mortality. (2) The results of recent studies have shown that the use of steroids before the birth of infants causes fetal lung maturation and may influence fetal biophysical activities. (3)

According to (4), measurable biophysical characteristics include breathing, movement, fetal tone, and the nonstress test (NST) of the fetal heart rate (FHR).

The administration of antenatal corticosteroids may change FHR and behavior that return to the baseline by four to seven days. (5) Several studies evaluated the effect of betamethasone and dexamethasone on FHR patterns and biophysical activities. (6-9) Studies reported that corticosteroid injection decreases biophysical profile (BPP) score although this is not a consistent finding. A nonreactive NST or low BPP score, which transiently occurs after corticosteroid administration, can mimic fetal distress, and thus clinicians need to be aware of this phenomenon in order to avoid unnecessary delivery. To the best of our knowledge, there is no systematic review and meta-analysis to summarize the results of these studies. Therefore, this systematic review and metaanalysis aimed at evaluating the effect of corticosteroids on BPP parameters.

\section{Methods}

Published studies until January 2018 were collected for a systematic review in order to evaluate the effect of corticosteroids (i.e., betamethasone and dexamethasone) on the BPP score and components by searching several databases such as PubMed/MEDLINE, Scopus, EMBASE, Cochrane Library, ISI Web of Science, Proquest, and Google scholar. Iranian databases including Scientific Information Database, Iranian Research Institute for Information Science and Technology, Magiran, IranMedex, and Barakat knowledge network system were searched as well. To this end, keywords were selected based on Mesh terms and included (but not limited to) "preterm delivery AND betamethasone OR dexamethasone OR fetal corticosteroid therapy AND biophysical profile OR Fetal heart rate variability OR FHR baseline OR nonstress test OR fetal behavior OR fetal breathing OR fetal tone OR fetal movement OR amniotic fluid". Moreover, an

Received 17 March 2019, Accepted 14 January 2020, Available online 15 February 2020

${ }^{1}$ Women's Reproductive Health Research Center, Tabriz University of Medical Sciences, Tabriz, Iran. ${ }^{2}$ Tabriz Health Service Management Research Center, Tabriz University of Medical Sciences, Tabriz, Iran. ${ }^{3}$ Iranian Center for Evidence-based Medicine, Tabriz University of Medical Sciences, Tabriz, Iran. ${ }^{4}$ Department of Physiology, Tabriz University of Medical Sciences, Tabriz, Iran. ${ }^{5}$ Maternal and Childhood Obesity Research Center, Urmia University of Medical Sciences, Urmia, Iran.

*Corresponding Author: Shabnam Vazifekhah, Email: shabnam.vazifekhah@yahoo.com 
overview of recent studies was carried out electronically and manually.

The criteria for reviewing articles related to this systematic study included the presence of two researchers who separately gathered the data. Moreover, the initial analysis of the results was performed by a single analyst and additional and irrelevant data were omitted accordingly.

Furthermore, prospective studies in English or Persian language were evaluated, including those that surveyed the effect of corticosteroids (betamethasone and dexamethasone) on FHR variability and baseline, BPP score and parameters, and fetal behavior and were published until January 2018. On the other hand, review articles, studies in other languages rather than Persian and English, articles with poor quality or related to organizations' reports, and letters to the editor, abstracts presented at conferences, thesis, or suggestions were excluded from this systematic review.

Data Extraction and Quality Assessment

Data characteristics related to the age of participation, demographic characteristics, and results were gathered and evaluated by two raters, and variations and points of the dispute were reviewed by the third rater.

Additionally, the selected articles for retrieval were assessed for methodological validity using Joanna Briggs Institute (JBI) Critical Appraisal Checklist for QuasiExperimental Studies (non-randomized experimental studies), the details of which are presented in Table S1 (see Supplementary file 1). The reviewers were blind to the results of each other's critical appraisal before conducting their own.

\section{Statistical Analysis}

Using Q statistic and $\mathrm{I}^{2}$, the heterogeneity level was determined and a significant level of $P<0.01$ was obtained for Cochran's Q test or $\mathrm{I}^{2}>50$ (10). In addition, publication bias was examined using the funnel plot and rank correlation test. Moreover, three methods proposed by Begg and Mazumdar were used to study if the administration of betamethasone and dexamethasone caused a reduction in BPP.

\section{Results}

Search Result and Study Characteristics

After examining related sites, 1068 articles were identified as the materials. Afterward, duplicated articles were eliminated with considering the selection criteria, and finally, only 56 research papers were opted for as the corpus of the study.

The methods of searching and selecting the articles are presented in Figure 1. Moreover, the basic characteristics of the study including the mean of depression and anxiety scores are shown in Table 1 (6-9, 11-18).

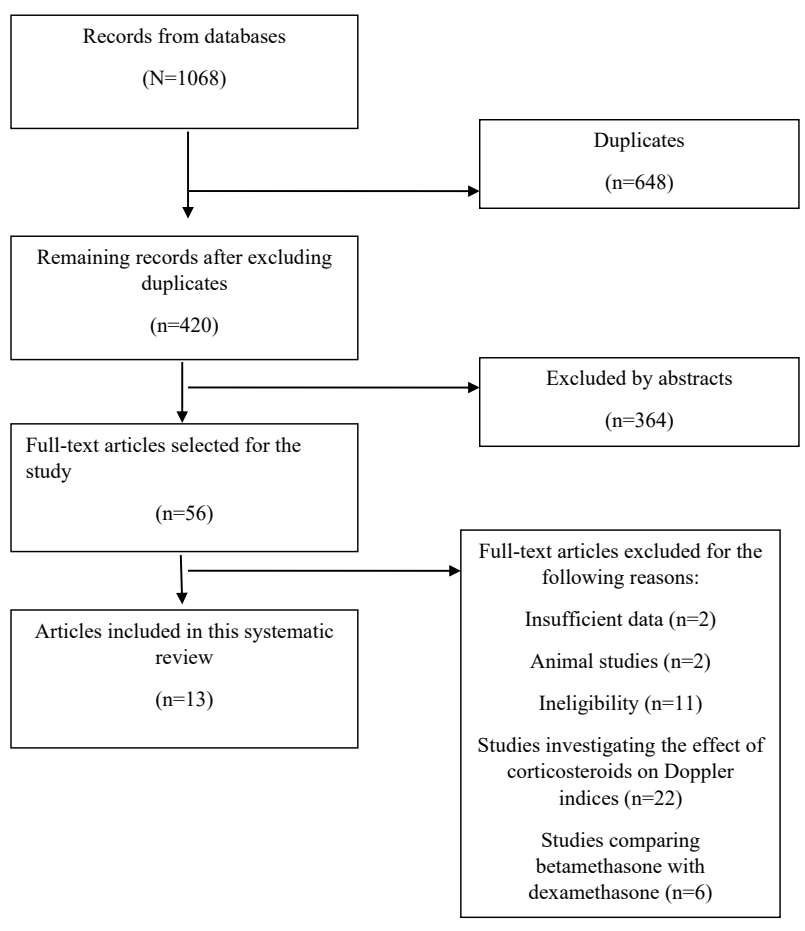

Figure 1. Flow Diagram for Article Selection.

\section{Participant Characteristics}

In selected studies, 490 pregnant women were enrolled who were at an increased risk for preterm delivery and received corticosteroid (betamethasone or dexamethasone) intramuscularly. Before and after injection, all five BPP markers were checked for any change and steroid effects were evaluated between 25 and 34 weeks of gestation. Then, the corticosteroid effect on the biophysical score lasted for 4-5 days.

Meta-analysis for the Effects of Corticosteroids on Biophysical Score

The random-effect model was used according to the identification of statistical heterogeneity. The forest plot is shown in Figure 2. The pooled event rates for the reduced amniotic fluid volume were $4.5 \%$ (95\% CI $=0.01$ 64.3, $P=0.1$ ), 76.8\% (95\% CI=33.5-95.6, $P=0.21)$, $71.8 \%$ (95\% CI $=38.8-91.1, P=0.18), 70.9 \%$ (95\% CI $=38.4-90.5$, $P=0.20$ ), and $92.3 \%$ (95\% CI $=76.0$ to $97.8, P<0.001)$ for baseline FHR, fetal breathing, fetal movement, and heart rate variability, respectively. However, the fetal tone was not reported in the selected studies.

\section{Quality of Studies}

As shown in Table S1, the quality assessment of the included studies was performed using JBI Critical Appraisal Checklist for Quasi-Experimental Studies. Based on the checklist, no bias was found in the included studies although no study had a control group. All studies were rated as high quality. 


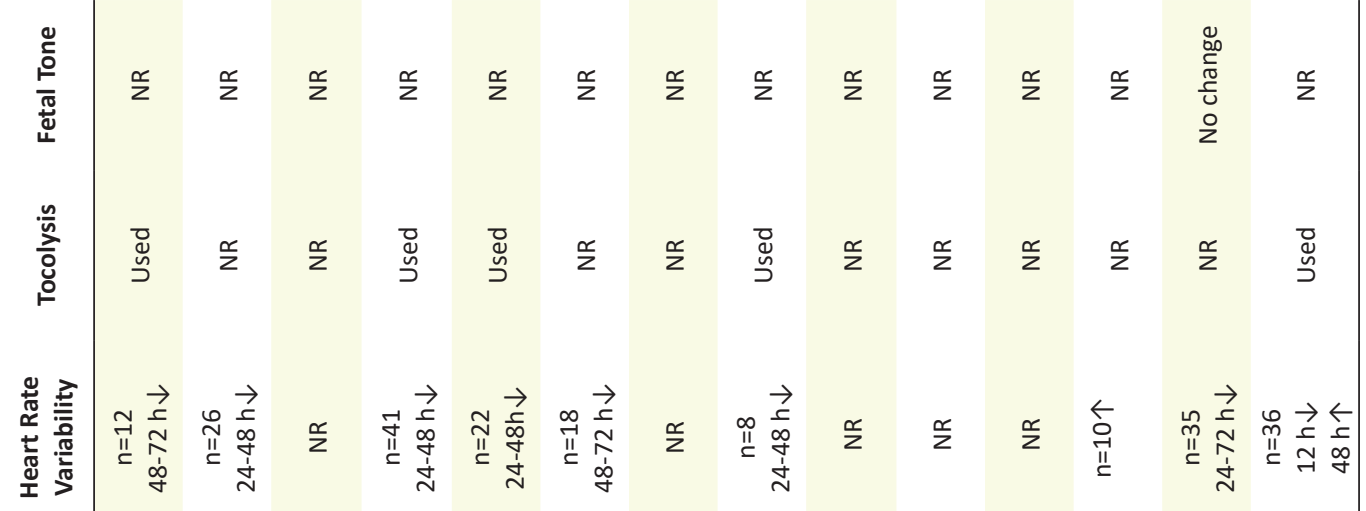

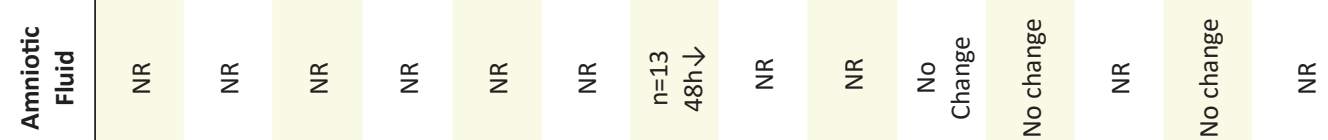

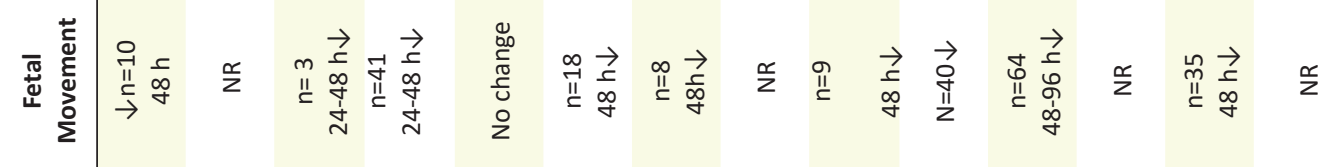

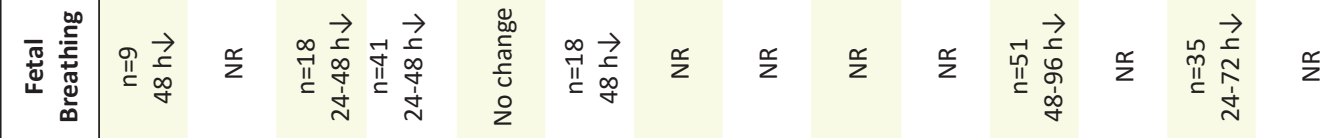

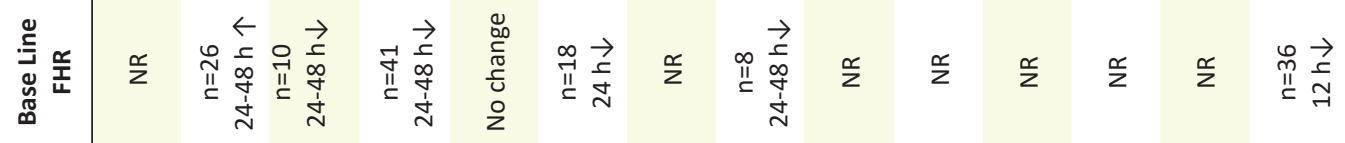

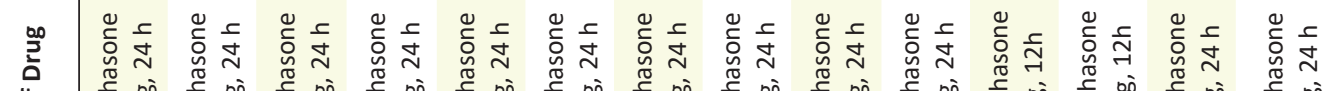

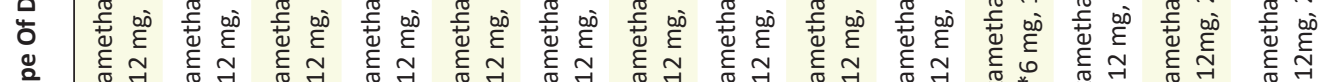

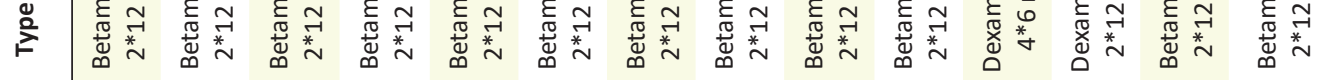

范

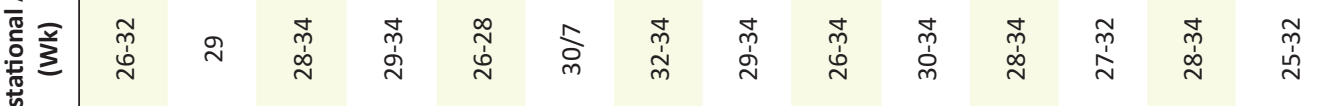

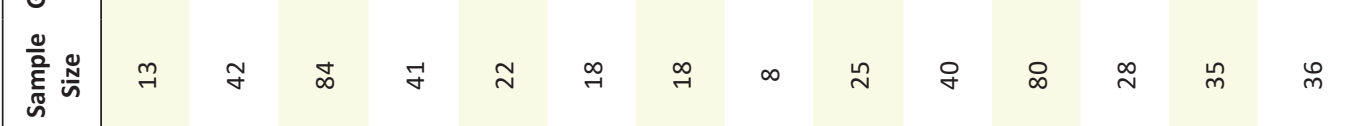

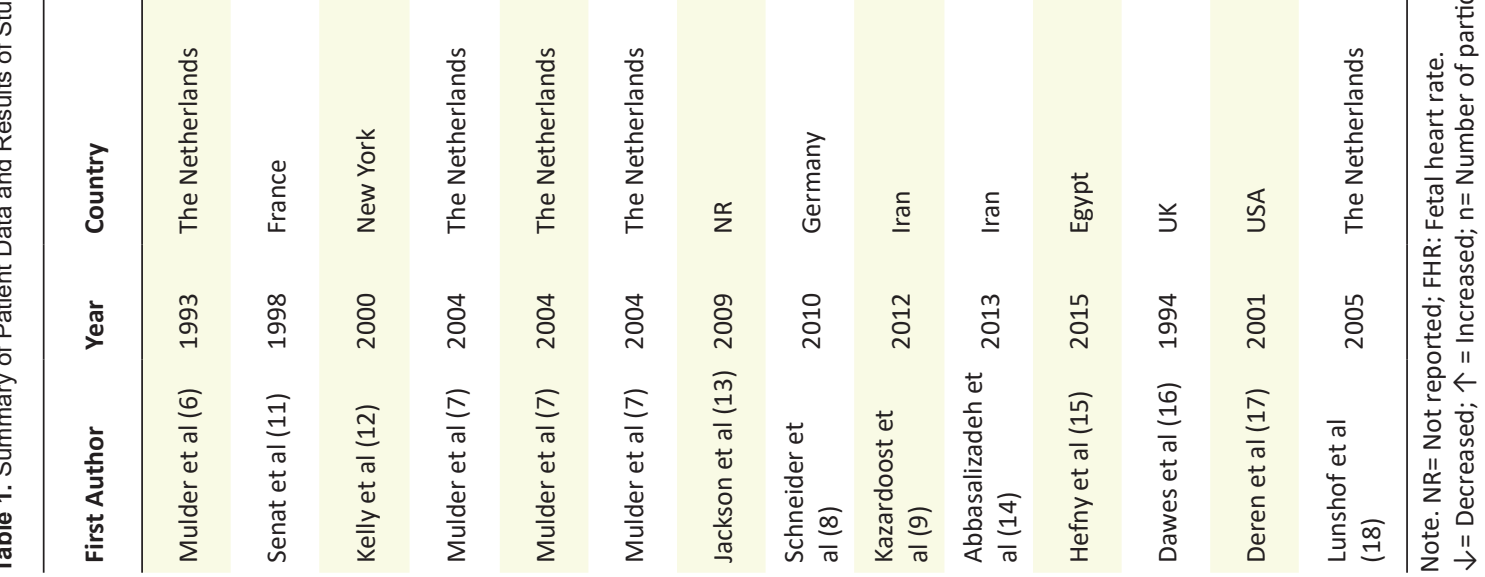




\section{Biophysical Profile}

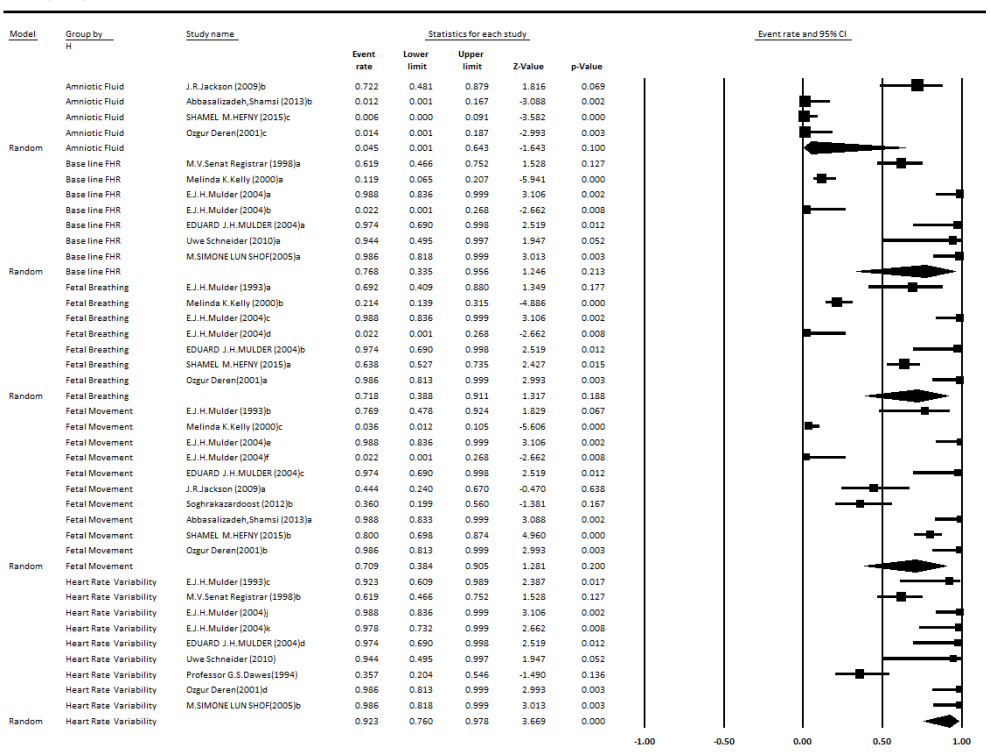

Figure 2. Forest Plot Showing the Pooled Event Rate for Biophysical Profile Parameters

Note. Studies are represented as squares, and the area of the square represents the weight given to the study in meta-analysis by CMA software. The overall event rate was calculated by a random-effects model. The diamond displays the overall estimated event rate and its $95 \%$ confidence interval.

\section{Discussion}

Preterm delivery causes 7\%-10\% of complications (17) and preterm birth is the cause of death and disability. According to Hefny et al (16), preterm birth is a risk factor for the respiratory distress syndrome (RDS), intraventricular hemorrhage (IVH), necrotizing enterocolitis (NEC), and patent ductus arteriosus (PDA) as well. To evaluate the effects of corticosteroids on biophysical parameters, a systematic and meta-analysis study was performed and the results of this fetal movement and fetal breathing, baseline FHR studies showed that betamethasone and dexamethasone decreased fluid volume although these changes were not significant. Notably, both steroids caused a significant decrease in the heart rate.

Corticosteroids (betamethasone and dexamethasone) are usually used in mothers who are likely to have preterm delivery in order to accelerate fetal maturation. (7) The effects of betamethasone on FHR and its general conditions at the prenatal stage depends on the age of pregnancy. In addition, corticosteroids are recommended at 24 to 34 weeks when the mother has to go into delivery for 7 days (7). Further, betamethasone and dexamethasone are potent drugs, which cross through the placenta and reach the fetus 2-3 hours after administration (7). Similarly, corticosteroid administration promotes surfactant secretions and increases fetal lung maturity (19). The results of various studies have shown that the administration of these two corticosteroids significantly reduces death, RDS, IVH, NEC, and PDA during pregnancy (15). The National Institute of Child Health and Human Development consensus panel in 2000 recommended a single course of corticosteroid for mothers at the risk of preterm delivery between 24 and 34 weeks within 7 days (20). A course of betamethasone consists of two $12 \mathrm{mg}$, intramuscular (IM) doses administered 24 hours apart. Furthermore, a dexamethasone course contains four $6 \mathrm{mg}$, IM doses administered 12 hours apart (21). Nonetheless, betamethasone seems to be superior in controlling RDS and other prematurity complications (22). Corticosteroids have been used for more than 30 years, and their side effects have been reported when used repeatedly $(19,22)$. Previous studies demonstrated that corticosteroid administration reduces fetal movements, fetal breathing, and FHR reactivity and thus decreases the BPP score (17). On the other hand, Deren et al (17) showed that corticosteroid administration did not change the amniotic fluid index and fetal tone although fetal breathing, fetal movements, FHR reactivity, and thus BPP score decreased up to 72 hours after corticosteroid administration. In another study, Ghasemi Tehrani et al (23) reported that amniotic fluid index did not change significantly after the administration of both betamethasone and dexamethasone, but both of these drugs reduced FHR reactivity significantly. Similarly, the results of our systematic review showed a significant decrease in FHR variability. The corticosteroid effect on BPP parameters is transient and returns to normal after several days $(6,17)$.

In contrast to betamethasone, dexamethasone has lower effects. In a previous study, dexamethasone made fewer changes in biophysical markers. On the other hand, there were more RDS, preterm delivery, and admission to the neonatal intensive care unit in the dexamethasone group (24). Likewise, Dawes et al (16) reported increased variability after dexamethasone administration. However, fetal movements and fetal breathing reductions were 
more common after the administration of betamethasone compared with dexamethasone (20).

Although the results of some studies showed an increase in FHR, there have been very few changes to justify the simultaneous decrease in variability although no change was reported in the morning. Conversely, FHR variability and fetal movements reduced in the afternoon and evening, but these changes were negligible in the morning $(6,7,24,25)$. According to evidence, the evaluation of fetal status at the time of betamethasone administration and afterward should be performed consecutively in the morning (26). The findings of a large-scale study (21) are consistent with the results of this study. Both studies suggested that fetal status evaluations during and after betamethasone administration should be based on consecutive records in the morning. It should be noted that the gestational age at the time of administration may affect the fetal response to corticosteroids, especially in fetuses older than 30 weeks $(6,7,26)$. Mulder et al showed that the effect of betamethasone on fetal movements and breathing was more evident in 29-34-week fetuses compared with 26-28-week fetuses (7).

The underlying mechanism related to the suppression of the biophysical activities of steroids is still unclear. Moreover, the direct effect of betamethasone on glucocorticosteroid receptors (GRs) in the fetal brain is not proven yet.

The human fetal brain uses hypothalamus-pituitaryadrenal (HPA) to control daily rhythms, breathing, along with heart- and GR-based activities. GRs in the human fetal brain are involved in controlling diurnal and ultradian rhythms, breathing, the heart, and HPA axis activity (7). Additionally, GRs have a high tendency toward corticosteroids and their reactions and effects vary from hours to days. Further, their functions suppress neural activity and interfere with many neurotransmitter systems and their complex transmission mechanisms (22).

Our systematic review revealed a significant reduction in FHR reactivity after corticosteroid administration. FHR variability is one of the fifth parameters in the BPP test. It is noteworthy that variability decreases in response to hypoxia. Furthermore, decreased variability after corticosteroid administration and its effect on the BPP score may be misinterpreted as fetal hypoxia and lead to unnecessary delivery of a preterm fetus. Accordingly, this effect of corticosteroids can help correctly interpret the BPP test (17). Kazardoost et al (9) showed that although the middle cerebral artery pulsatility index did not decrease after corticosteroid administration, it helped distinguish this effect of corticosteroids from hypoxia. Although the Doppler study can help differentiate fetal hypoxia from the corticosteroid-induced decreased BPP score, future studies are needed to confirm this role of Doppler and prevent unnecessary preterm deliveries when the BPP score decreases after corticosteroid administration.

\section{Benefits and Limitations of the Study}

The main strength of our systematic study was that it used no artificial data. In addition, methods and data analysis of this study were based on predefined criteria and were completed and controlled using well-designed instruments. Finally, data were reviewed independently by two raters.

\section{Conclusions}

This study has some limitations. Publication bias could not be excluded (i.e., negative findings are less likely to be published). Nonetheless, a significant decrease was observed in heart rate variability, which was a transient effect and returned to a normal level after several days. Further studies are needed to distinguish fetal distress from the effect of corticosteroids on the BPP score (Doppler study). Eventually, many studies are required to identify the effect of steroids on the adaptation of fetal biophysical parameters.

\section{Conflict of Interests}

The authors have no conflict of interests relevant to this article.

\section{Ethical Issues}

Not applicable.

\section{Financial Support}

None.

\section{Acknowledgment}

We would like to appreciate the editors and referees for considering our study. In addition, special thanks go to Research Development and Cooperation Center and Iranian Center for Evidence-Based Medicine, Tabriz University of Medical Sciences for absolute support.

\section{Supplementary Materials}

Supplementary file 1 contains Table S1.

\section{References}

1. Effect of corticosteroids for fetal maturation on perinatal outcomes. NIH Consensus Development Panel on the Effect of Corticosteroids for Fetal Maturation on Perinatal Outcomes. JAMA. 1995;273(5):413-418. doi:10.1001/ jama.1995.03520290065031

2. ACOG practice bulletin no. 127: management of preterm labor. Obstet Gynecol. 2012;119(6):1308-1317. doi:10.1097/ AOG.0b013e31825af2f0

3. Rotmensch S, Liberati M, Celentano C, et al. The effect of betamethasone on fetal biophysical activities and Doppler velocimetry of umbilical and middle cerebral arteries. Acta Obstet Gynecol Scand. 1999;78(9):768-773.

4. Lalor JG, Fawole B, Alfirevic Z, Devane D. Biophysical profile for fetal assessment in high risk pregnancies. Cochrane Database Syst Rev. 2008;2008(1):CD000038. doi:10.1002/14651858.CD000038.pub2 
5. Verdurmen KM, Renckens J, van Laar JO, Oei SG. The influence of corticosteroids on fetal heart rate variability: a systematic review of the literature. Obstet Gynecol Surv. 2013;68(12):811-824. doi:10.1097/ogx.0000000000000002

6. Mulder EJ, Derks JB, Zonneveld MF, Bruinse HW, Visser GH. Transient reduction in fetal activity and heart rate variation after maternal betamethasone administration. Early Hum Dev. 1994;36(1):49-60. doi:10.1016/03783782(94)90032-9

7. Mulder EJ, Koenen SV, Blom I, Visser GH. The effects of antenatal betamethasone administration on fetal heart rate and behaviour depend on gestational age. Early Hum Dev. 2004;76(1):65-77. doi:10.1016/j.earlhumdev.2003.10.007

8. Schneider U, Fiedler A, Schröder B, et al. The effect of antenatal steroid treatment on fetal autonomic heart rate regulation revealed by fetal magnetocardiography (fMCG). Early Hum Dev. 2010;86(5):319-325. doi:10.1016/j. earlhumdev.2010.05.018

9. Kazardoost S, Pooransari P, Mirzamoradi M, Hashemi Mohammad Abad Nazir. The effect of betamethasone on fetal movement, biophysical profile and fetal circulation in preterm fetuses. Life Sci J. 2012;9(4):354-356.

10. Higgins JP, Green S. Cochrane Handbook for Systematic Reviews of Interventions. John Wiley \& Sons; 2011

11. Senat MV, Minoui S, Multon O, Fernandez H, Frydman $\mathrm{R}$, Ville Y. Effect of dexamethasone and betamethasone on fetal heart rate variability in preterm labour: a randomised study. Br J Obstet Gynaecol. 1998;105(7):749-755. doi:10.1111/j.1471-0528.1998.tb10206.x

12. Kelly MK, Schneider EP, Petrikovsky BM, Lesser ML. Effect of antenatal steroid administration on the fetal biophysical profile. J Clin Ultrasound. 2000;28(5):224-226. doi:10.1002/ (sici)1097-0096(200006)28:5<224::aid-jcu3>3.0.co;2-g

13. Jackson JR, Kleeman S, Doerzbacher M, Lambers DS. The effect of glucocorticosteroid administration on fetal movements and biophysical profile scores in normal pregnancies. J Matern Fetal Neonatal Med. 2003;13(1):5053. doi:10.1080/jmf.13.1.50.53

14. Abbasalizadeh S, Neghadan Pharabar Z, Abbasalizadeh F, Ghojazadeh M, Goldust M. Efficacy of betamethasone on the fetal motion and biophysical profile and amniotic fluid index in preterm fetuses. Pak J Biol Sci. 2013;16(22):15691573. doi:10.3923/pjbs.2013.1569.1573

15. Hefny SM, Hashem AM, Yehia AE. Effects of Maternal Corticosteroids Administration for Enhancement of Fetal Lung Maturity on Non Stress Test and Fetal Biophysical Activities. Med J Cairo Univ. 2015;83:43-46.

16. Dawes GS, Serra-Serra V, Moulden M, Redman CW. Dexamethasone and fetal heart rate variation.
Br J Obstet Gynaecol. 1994;101(8):675-679. doi:10.1111/j.1471-0528.1994.tb13183.x

17. Deren O, Karaer C, Onderoglu L, Yigit N, Durukan T, Bahado-Singh RO. The effect of steroids on the biophysical profile and Doppler indices of umbilical and middle cerebral arteries in healthy preterm fetuses. Eur J Obstet Gynecol Reprod Biol. 2001;99(1):72-76. doi:10.1016/s03012115(01)00367-0

18. Lunshof MS, Boer K, Wolf $\mathrm{H}$, Koppen S, Velderman JK, Mulder EJ. Short-term $(0-48 \mathrm{~h})$ effects of maternal betamethasone administration on fetal heart rate and its variability. Pediatr Res. 2005;57(4):545-549. doi:10.1203/01. pdr.0000155948.83570.eb

19. Niroomanesh S, Shojaei K, Moghadam SF, Mohammadi N, Rahimi Z, Rezaei Keyhanaei K. Effect of prenatal betamethasone on fetal, uteroplacental, and maternal blood flow velocity in pregnancies complicated by fetal growth restriction. Int J Gynaecol Obstet. 2015;130(3):270-273. doi:10.1016/j.ijgo.2015.04.043

20. Gabbe SG, Niebyl JR, Simpson JL, et al. Obstetrics: Normal and Problem Pregnancies E-Book. Elsevier Health Sciences; 2016.

21. de Heus R, Mulder EJ, Derks JB, Koenen SV, Visser GH. Differential effects of betamethasone on the fetus between morning and afternoon recordings. J Matern Fetal Neonatal Med. 2008;21(8):549-554. doi:10.1080/14767050802128214

22. Mulder EJ, de Heus R, Visser GH. Antenatal corticosteroid therapy: short-term effects on fetal behaviour and haemodynamics. Semin Fetal Neonatal Med. 2009;14(3):151-156. doi:10.1016/j.siny.2008.10.003

23. Ghasemi Tehrani H, Khani B, Komrani Z. Comparison of the effect of betamethasone versus dexamethasone on the amniotic fluid index in the women at risk of preterm labor. J Res Med Sci. 2014;19(12):1124-1128.

24. Subtil D, Tiberghien P, Devos P, et al. Immediate and delayed effects of antenatal corticosteroids on fetal heart rate: a randomized trial that compares betamethasone acetate and phosphate, betamethasone phosphate, and dexamethasone. Am J Obstet Gynecol. 2003;188(2):524-531. doi:10.1067/ mob.2003.136

25. Rotmensch S, Lev S, Kovo M, et al. Effect of betamethasone administration on fetal heart rate tracing: a blinded longitudinal study. Fetal Diagn Ther. 2005;20(5):371-376. doi:10.1159/000086815

26. Koenen SV, Mulder EJ, Wijnberger LD, Visser GH. Transient loss of the diurnal rhythms of fetal movements, heart rate, and its variation after maternal betamethasone administration. Pediatr Res. 2005;57(5 Pt 1):662-666. doi:10.1203/01.pdr.0000159762.50504.1f

(c) 2020 The Author(s); This is an open-access article distributed under the terms of the Creative Commons Attribution License (http:// creativecommons.org/licenses/by/4.0), which permits unrestricted use, distribution, and reproduction in any medium, provided the original work is properly cited. 